\title{
KAPITEL 2
}

Beata Grzeszczakowska-Pawlikowska*

\section{Germanistikabsolventen/-innen am Servicedesk - Chancen oder Schwierigkeiten für das Fach?}

\section{Einleitende Bemerkungen}

Die Ausbildung zum(r) Deutschlehrer/-in war für jede(n) polnische(n) Germanistikstudenten/-in über lange Jahre hinweg, insbesondere während des großen Booms für das Fremdsprachenlernen nach 1989, eine Selbstverständlichkeit. Lediglich vereinzelt lehnten Studierende damals die sichere Lehrerexistenz bewusst ab. Diese Zeiten scheinen mittlerweile endgültig vorbei zu sein. Die Lehrveranstaltungen zur Didaktik und Methodik des Deutschunterrichts werden aktuell von einer immer kleineren Zahl von Studierenden belegt, sodass manche DaF-Module gar nicht stattfinden. Für diesen Trend lassen sich einige miteinander eng verwobene Gründe anführen. Zunächst sind es die seit langem schon europa- bzw. weltweit zu verzeichnende Vorherrschaft des Englischen und der daraus resultierende hohe Stellenwert dieser Sprache als 1. obligatorische Fremdsprache in der schulischen Ausbildung. Hinzu kommt das demografische Tief mit seinen weitreichenden Folgen für das Bildungssystem, wie etwa die Vergrößerung von Klassen bzw. die Schließung von Schulen bzw., vor allem in den strukturschwächeren Gebieten Polens. Diese beiden Faktoren liegen der stets sinkenden Nachfrage u.a. nach DaF-Lehrkräften im Schulbereich zugrunde.

*Universität Łódź, Institut für Germanistik. 
Schließlich spielen in diesem Zusammenhang ebenso ökonomische Aspekte eine wesentliche Rolle. Zu betonen ist diesbezüglich vorrangig die wachsende Diskrepanz zwischen der finanziellen Misere der polnischen Lehrenden und den verhältnismäßig hohen Gehältern im Wirtschaftssektor. Die Lehrpersonen sehen sich folglich gezwungen, außer ihrer Anstellung an Schulen verschiedenen Nebentätigkeiten nachzugehen. Gleichzeitig werden den Germanistikabsolventen/-innen bereits am Karrierestart, neben vielen Sozialleistungen und Entwicklungsmöglichkeiten, attraktive Verdienstbedingungen angeboten. Es verwundert also nicht, dass sich stets mehr angehende Germanisten/-innen als Spezialisten/-innen in verschiedenen Branchen der Wirtschaft beruflich verwirklichen wollen. Dies umso mehr, als der Bedarf an Mitarbeiter/-innen mit Deutschkenntnissen derzeit durchaus hoch.

Vor diesem Hintergrund stellt sich nun die Frage, was die bestehenden Umstände auf dem Arbeitsmarkt konkret für die polnische Germanistik bedeuten. Sind sie als Chancen für die Neuorientierung des Faches zu werten, das aktuell eigene traditionell etablierte Ausrichtung zu bewahren sucht, oder vielmehr als Schwierigkeiten zu betrachten, auf welche die (noch) traditionell sozialisierten Hochschullehrer/-innen der philologischen Fachrichtungen möglicherweise stoßen? Dieser Fragestellunger widmet sich der vorliegende Beitrag. Ausgegangen wird zunächst von einigen quantitativen und qualitativen Angaben über die Nutzung von Fremdsprachen im polnischen Wirtschaftsumfeld. In den Fokus weiterer Ausführungen rücken exemplarisch Aufgaben, welche im Servicedesk eines internationalen Konzernes in Łódź von polnischen Germanistikstudierenden bzw. gebildeten Germanisten/-innen in der Fremdsprache Deutsch zu bewältigen sind.

\section{Nutzung von Fremdsprachen im Wirtschaftssektor}

Der Wirtschaftssektor, in dem die polnischen Germanistikabsolventen/-innen am häufigsten Anstellung finden, lässt sich zusammenfassend als Sektor moderner Dienstleistungen bezeichnen. Dazu gehören BPO- (Business Process Outsorcing-), SSC- (Shared-Services-), ITO- (Information Technology Outsorcing-) und R\&D- (Research Development-)Zentren. Im Großen und Ganzen kommt es dabei auf das Outsorcing bzw. Sharing an externe bzw. interne (innerhalb eines Großunternehmens) Provider an, d.h. das Auslagern ganzer Support-/Unterstützungsprozesse, die selbst keinen direkten Kundennutzen erzeugen und deshalb nicht wettbewerbsdifferenzierend sind. Das Auslagern bzw. Sharing mancher Geschäftsprozesse erlaubt den Firmen eine Effizienzsteigerung, indem sie sich auf das Hauptgeschäft, sog. Kernprozesse (z.B. Produktionsprozesse) als Tätigkeiten konzentrieren können, 
die der Wertschöpfung des Unternehmens dienen. Die mögliche Aufgabenauslagerung bzw. deren Sharing erfolgt vornehmlich in folgenden Bereichen: Finanz- und Rechnungswesen, Buchhaltung, Bankwesen, Personalwesen, Beschaffung und Logistik, Customer Care/IT, Erstellung technischer Dokumentation und Übersetzungen, Ausbildung und Training, Entwicklung und Forschung (vgl. Włodarczyk 2016; Wassermann 2008).

Der letzte ABSL-Bericht (2018) hebt in diesem Zusammenhang zum einen den landesweit systematischen Anstellungszuwachs in den modernen Dienstleistungen hervor, zum anderen die gezielte Erweiterung der dort bereits vorhandenen Tätigkeitsbereiche ${ }^{1}$. Im 1. Jahresviertel 2018 gab es in Polen insgesamt 1236 polnische und ausländische BPO-, SSC, IT- bzw. R\&D-Zentren mit insgesamt 279000 Mitarbeiter/-innen. Im Zeitraum vom Januar 2017 bis Ende März 2018 nahmen 91 neue Unternehmen ihre Tätigkeit auf - seit Januar 2016 werden in diesem Bereich um 30\% Arbeitsstellen mehr angeboten. Wie jedes Jahr dominieren unter den neuen Investoren ausländische Firmen (86\%). Die größten Zentren für moderne Dienstleistungen sind in folgenden Städten ansässig: Kraków, Warszawa, Wrocław (TIER 1), weiterhin im sog. Trójmiasto (Gdańsk, Gdynia, Sopot), in der Agglomeration von Katowice, Łódź und Poznań (TIER 2) mit jeweils $40 \%$ neuen Investitionen und nicht zuletzt in Bydgoszcz, Lublin, Rzeszów und Szczecin (TIER 3) mit 20\% neuen Firmen ${ }^{2}$. Nach optimistischen Prognosen sollen bis zum Jahr 2020340000 neue Arbeitsplätze in diesem Bereich entstehen (vgl. ABSL-Bericht 2018, 6f.). Zu den wichtigsten Partnern, die von den modernen Dienstleistungszentren in Polen bedient werden, gehören folgende Länder: Deutschland, Großbritannien, die USA, Polen und Frankreich, gefolgt von der Schweiz, Dänemark, den Niederlanden, Schweden, Belgien, Finnland, Spanien, Norwegen, Italien und Russland. $48 \%$ der in Polen ansässigen Zentren bieten ihre Dienstleistungen weltweit an (vgl. ebd.: 39).

Der Einsatz zahlreicher Sprachen zur mündlichen und schriftlichen Kundenkommunikation ist die logische Konsequenz dieser Entwicklungen auf dem polnischen Arbeitsmarkt. Die meist nützlichen Sprachen, die in den BPO-, SSC-, ITO- und R\&D-Zentren im Jahr 2018 regelmäßig in Anwendung kamen, sind nach aktuellen ABSL-Angaben Englisch in $99 \%$ aller Unternehmen, Polnisch in entsprechend 88\%, Deutsch in 72\%, Französisch in 59\%, Italienisch in 52\% und Spanisch in 50\% aller Unternehmen. Zu weiteren Sprachen, in denen mit Kunden täglich kommuniziert wurde, gehören:

\footnotetext{
${ }^{1}$ ABSL steht für den Verein von Leadern der Business-Dienstleistungen.

${ }^{2}$ Bei TIER handelt es sich laut dem ABSL-Bericht (2018) um einen Indikator für die Beschäftigungsgröße.
} 
Niederländisch in entsprechend 35\% aller Unternehmen, Russisch in 34\%, Portugiesisch in 28\%, Tschechisch in 25\%, Schwedisch, Dänisch sowie Norwegisch in jeweils 24\% der Firmen. In bis 20\% der Dienstleistungszentren werden nicht zuletzt auch Ungarisch, Finnisch, Ukrainisch, Slowakisch, Türkisch, Rumänisch, Bulgarisch, Arabisch, Griechisch, Kroatisch, Hebräisch, Litauisch, Lettisch, Slowenisch, Chinesisch, Serbisch, Estnisch, Japanisch, Weißrussisch als Kundensprachen gebraucht, sowie vereinzelt auch Hindi, Koreanisch, Urdu (vgl. ebd.: 41). Laut der ABSL-Erhebung für das Jahr 2017 waren für 68\% der Befragten Deutschkenntnisse besonders wichtig, für die Mehrzahl der befragten Dienstleistungszentren (64\%) war Deutschland der wichtigste Kunde. Gefragt waren ferner Französisch, nordische Sprachen und Niederländisch - von entsprechend 34\%, 31\% und 22\% der untersuchten Unternehmen. Als am häufigsten von modernen Dienstleistungszentren geforderte Kompetenzen galten 2017 als Top 1 Fremdsprachenkenntnisse allgemein sowie Deutschkenntnisse auf dem Niveau C1 (neben dem Fachwissen im Software-Bereich), als Top 2 Französisch- und Englischkenntnisse (neben z.B. dem Fachwissen im SAP- und Java-System sowie im Rechnungswesen) und als Top 3 Kenntnisse des Tschechischen (u.a. neben beruflicher Erfahrung allgemein) (vgl. ABSL-Bericht 2017: 33f.). Nach den aktuellen ABSL-Angaben werden in einer Firma im Umgang mit Kunden durchschnittlich acht Sprachen verwendet, in 29\% aller BPO-, SSC-, ITObzw. R\&D-Zentren wird dabei in mindestens zehn Sprachen kommuniziert, der absolute Rekord sind 35 Sprachen in ein und demselben Unternehmen (vgl. ABSL-Bericht 2018: 41).

Deutsch und Französisch sind nach Englischkenntnissen als obligatorischer Anforderung der Arbeitgeber/-innen ebenfalls in den Firmen in Łódź die meist verwendeten Fremdsprachen. Dies ergab eine Erhebung zur Fremdund Fachsprachenkommunikation im Wirtschaftsumfeld von Makowski (2018), welche im Zeitraum vom 15.08. bis zum 15.10.2017 als Teil des Forschungsprojekts Lodzer Sprachbarometer (laufend seit 2014 an der Philologischen Fakultät der Universität Łódź) stattfand. An der Befragung nahmen vier von den insgesamt 70 in der Stadt und ihrer Umgebung ansässigen BPO-, SSC-, ITO- und R\&D-Zentren mit 5506 Mitarbeiter/-innen (30,4\% von rund 18100) teil (ausführlicher dazu s. Angaben des ABLS-Berichts 2017). Daten zur Frage nach der Nutzung von Fremdsprachen lieferten drei der vier Unternehmen mit einer gesamten Beschäftigungsquote von 3606 Mitarbeiterin/-innen. Im Einzelnen galt Deutsch für 371 Mitarbeiterin/-innen und Französisch für 295 Mitarbeiterin/-innen als führende Verkehrssprache in Geschäftskontakten. Zu weiteren, in diesem Kontext benutzten Fremdsprachen gehören Spanisch, Italienisch, Russisch, Niederländisch, Portugiesisch und Rumänisch 
- sie werden entsprechend von 132, 90, 70, 36, 30 und 21 Mitarbeiter/-innen alltäglich verwendet. Laut Makowski (2018) manifestiert sich dieser Trend direkt in der Nachfrage der Arbeitgeber/-innen nach konkreten Kompetenzen der Jobsuchenden. Zum Zeitpunkt der Befragung waren Englisch und Deutsch am dringendsten gesucht - mit entsprechend 85 und 68 offenen Stellen, gefolgt von Französisch mit 32 offenen Stellen, Spanisch und Italienisch mit je 17 offenen Stellen sowie Russisch mit 10 offenen Stellen (ausführlicher vgl. dazu Makowski 2018; Makowski in diesem Sammelband).

Im Hinblick auf die von den BPO-, SSC-, ITO- und R\&D-Zentren bedienten Prozesskategorien lässt sich insgesamt eine starke Heterogenität verzeichnen, indem weltweit ein breites Spektrum von Dienstleistungen angeboten wird. $\mathrm{Zu}$ den meist bedienten Branchen gehören u.a. das Bank- und Finanzwesen, Versicherungen (BFSI), Informationstechnologie, Personalwesen (HR Services), juristische Dienstleistungen (Legal Services) sowie Forschung und Beratung (Research \& Consulting Services) (vgl. ABLS-Bericht 2018: 37). Dies trifft ebenfalls für die in Łódź ansässigen Unternehmen zu, die Aufträge werden hier vor allem in folgenden Bereichen realisiert: Finanzen und Buchhaltung, Personalmanagement, Kundenbetreuung (in allen vier befragten Unternehmen), ferner Bankwesen, Finanzdienstleistungen und Versicherung und Dokumenten- und Projektmanagement (in drei von vier befragten Unternehmen). Alle vier befragten Firmen planten, den jeweiligen Tätigkeitsbereich zukünftig zu erweitern und somit das Personal auszubauen. Zur Abwicklung von Kundenaufträgen sind schließlich alle vier Sprachfertigkeiten im Deutschen als Fremdsprache notwendig. Dabei kommt es jeweils vor allem auf das Verständnis bzw. die Produktion von fachspezifischen Textsorten an, wie etwa (Telefon-)Gespräche, Anweisungen/Anleitungen/Erklärungen, Präsentationen, Diskussionen und Verhandlungen, (E-Mail-)Korrespondenz, Rechnungen, Informationen in der (Fach-)Presse, Notizen aus Sitzungen/Besprechungen. Hinzu kommt die translatorische Kompetenz, die in den modernen Dienstleistungszentren ebenfalls von Relevanz sein kann (ausführlicher vgl. dazu Makowski 2018; auch in diesem Sammelband).

\section{Tätigkeit im Servicedesk am Beispiel von Fujitsu - Aufgabenbereich und Schulungsbedarf}

Fujitsu Technology Solutions GmbH gehört neben Capita, CERI International, Infosys, Nordea Bank, UPS Global Business Services und vielen anderen (vgl. ABLS-Bericht 2018: 27) zu den führenden Zentren für moderne Dienstleistungen, die in Łódź tätig sind. Die Geschäftstätigkeit des Unternehmens 
umfasst vor allem Infrastrukturdienste, die Remote-Verwaltung der Infrastruktur als dritte Ebene des IT-Supports, Geschäftsanwendungsdienste, die Forschung und Entwicklung mit einem Softwareentwicklungszentrum, das SSC im Bereich der Finanzen und nicht zuletzt den Servicedesk als erste und zweite Ebene des IT-Supports ${ }^{3}$.

Bei Fujitsu Technology Solutions GmbH in Łódź findet hochqualifiziertes Personal mit sprachlichen und technischen Kompetenzen Anstellung. Im September 2017 hatten von den insgesamt 2147 Mitarbeiter/-innen ca. 80\% eine akademische Ausbildung. Am Service Desk arbeiteten zu diesem Zeitpunkt 1269 Personen, davon 250 mit Deutschkenntnissen als führender Sprache (und obligatorischen Englischkenntnissen). Deutsch ist im FujitsuServicedesk die nach Englisch am stärksten vertretene Sprache, mit großem Abstand zu anderen, wie Französisch, Spanisch, Russisch und Niederländisch $^{4}$. Die wichtigsten Kunden des Servicedesk-Teams in Łódź vertreten u.a. die folgenden Industriebereiche: Finanz-, Automobil-, Bau-, Telekommunikations- und Versorgungswesen.

Für eine Servicedesk-Stelle werden von Kandidaten/-innen neben Sprachkompetenzen auch grundlegende IT-Kenntnisse sowie Erfahrungen im Kundenservice erwartet. $\mathrm{Zu}$ den formalisierten Einarbeitungsprozeduren der neu angestellten Mitarbeiter/-innen gehören Schulungen zum Serviceangebot von Fujitsu Technology Solutions, IT-Basiswissen und zu Grundlagen in Kundenkommunikation, darunter zur schriftlichen Kommunikation und zum Zeitmanagement. Im Moment der Anstellung wird für jede(n) Mitarbeiter/-in ein sog. PDP, ein Personal Development Plan erstellt, nach dem fehlende Personal- bzw. fachbezogene Kompetenzen nachgeholt werden müssen. Zusätzlich umfasst der PDP einen Entwicklungspfad mit konkreten Einzelschritten (Stepping Stones) sowie einen zeitlichen Rahmenplan zur besseren Überprüfung der Fortschritte.

Zu Aufgaben der Servicedesk-Agenten bei Fujitsu Technology Solutions GmbH gehört die Kundenbetreuung im weitesten Sinne des Wortes, d.h. alle Aspekte der Vermittlung zwischen Kunde und Dienstleister. Folglich fungiert der Servicedesk als zentrale Anlaufstelle für einen strukturierten und kundenorientierten Umgang mit allen Anfragen von IT-System-Anwendern/-innen. Im Einzelnen kommt es dabei auf die Überprüfung, Verifizierung und Kategorisierung von eingehenden Problemmeldungen sowie deren weitere Bearbeitung an, indem eine angeforderte technische „erste Hilfe" erteilt wird

${ }^{3}$ In Łódź seit 2009 ansässig (Textorial Park, Fabryczna 17, 90-344 Łódź, Polen, www. pl. fujitsu.com).

${ }^{4} 870$ Fujitsu Servicedesk-Mitarbeiter/-innen sind allerdings ausschließlich des Englischen mächtig. 
bzw. schwerwiegendere IT-Probleme an die IT-Spezialisten/-innen weitergeleitet werden. Die Kundenbetreuung erfolgt sowohl mündlich (durch Telefongespräche) als auch schriftlich (durch E-Mails). Als weitere Aufgaben werden ebenfalls eine systematische Berichterstattung bezüglich der angemeldeten Kundenschwierigkeiten sowie eine entsprechende Dokumentationsführung allgemein definiert. Der Aufgabenbereich der Servicedesk-Experten umfasst u.a. die Unterstützung der Servicedesk-Agenten bei der Lösung der von Kunden angemeldeten Probleme, das Erstellen von Arbeitsanweisungen für den Umgang mit Kunden und möglichen IT-Problemen, die Aktualisierung der Datenbank, der sog. Knowledgebase, die Zusammenarbeit mit IT-Experten auf höheren Ebenen, die Koordination bei der Einführung neuer Dienstleistungen, die ständige Verbesserung der Qualität erteilter Dienstleistungen und die Bearbeitung von Schulungsplänen für neu angestellte Mitarbeiter/-innen bzw. deren regelmäßiges Training. Die Sicherung einer guten Atmosphäre am Arbeitsplatz gilt als spezielle Aufgabe der Teamleaders. Alle ServicedeskMitarbeiter/-innen sind nicht zuletzt verpfichtet, sich regelmäßig und systematisch im Bereich der modernen Informationstechnologien weiterzubilden.

Nach Aussagen der Fujitsu-Servicedesk-Mitarbeiter/-innen in Łódź gehören zu ihren Stärken am Arbeitsplatz u.a. die folgenden Fähigkeiten bzw. Fertigkeiten sowie Eigenschaften ${ }^{5}$ :

- gute Fremdsprachen- bzw. Deutschkenntnisse und ihre ständige Verbesserung;

- Vertrautheit mit IT-Verfahren sowie mit Aufgaben im Servicedesk-Bereich, darunter schnelle Diagnose der eingehenden IT-Probleme, Erfahrung bei der Lösung von IT-Problemen, Software-Kenntnisse, professionelle Dokumentationsführung;

- Fähigkeit zu einer schnellen Einarbeitung in neue Aufgaben und Prozesse sowie der systematische Wissenserwerb im IT-Bereich;

- analytische Fähigkeiten, analytisches Denkvermögen, Schlussfolgerungsfähigkeit allgemein;

- bewusstes, zielgerichtetes, professionelles, kundenorientiertes Handeln sowie die Entscheidungsfähigkeit;

- Teamfähigkeit und Personalmanagement;

- fristgerechte Aufgabenausführung, darunter gutes Zeit- und Aufgabenmanagement, gute Arbeitsorganisation, Fleiß, Ehrgeiz;

- höfliche Umgangsformen im direkten Umgang mit Kunden, Geduld und Verständnis gegenüber Kunden;

${ }^{5}$ Die Aussagen stammen aus einer Befragung, die im November 2017 unter Service Desk-Mitarbeiter/-innen mit Deutschkenntnissen in Fujitsu Technology GmbH Łódź durchgeführt wurden. Die Rücklaufquote belief sich auf 34 ausgefüllte Fragebögen. 
- ein angemessener Umgang mit stressigen Situationen, ggf. mit schwierigen Kunden.

Nach gewünschten Fortbildungsthemen gefragt, nannten die Servicedesk-Mitarbeiter/-innen vor allem die folgenden Bereiche:

- Verbesserung der Fremdsprachen-/Deutsch- bzw. Englischkenntnisse mit besonderem Fokus auf die Fertigkeit des Hörverstehens sowie die Fertigkeit des Sprechens zur effizienten Kundenbetreuung (zur optimalen Führung von Telefongesprächen);

- Vertiefung des IT-Fachwortschatzes, ggf. der Erwerb von IT-Fachsprache in der Zielsprache Deutsch;

- interpersonelle Kommunikation, u.a. der Erwerb der Fähigkeit zur konstruktiven Konfliktaustragung und zum professionellen Umgang mit Kunden;

- Personalmanagement;

- eigene Arbeitsorganisation, u.a. Zeitmanagement;

- IT-systembezogene Schulungen, u.a. im Bereich von Windows 10, Linux, Microsoft, MS-Office (Excel, Power-Point, Outlook);

- Training im Bereich der Analyse und Lösung von Netzwerkproblemen, systematische Erweiterung des allgemeinen IT-Fachwissens.

Zusammenfassend handelt es sich im Falle einer Anstellung im Servicedesk bei Fujitsu Technology Solutions GmbH Łódź um ein vielfältiges Aufgabenspektrum, welches nicht nur mit Hilfe von Fremdsprachenkenntnissen zu bewältigen ist. Zur Ausführung dieser Aufgaben sind ferner ebenfalls konkrete, fach-/IT-bezogene Kenntnisse notwendig. Eine wichtige Voraussetzung dafür stellen schließlich zahlreiche inter- und intrapersonelle Fähigkeiten, sog. soft skills dar. Die Fujitsu-Mitarbeiter/-innen scheinen sich dieser, für ihre Tätigkeit im Servicedesk erforderliche Kompetenzenvielfalt bewusst zu sein, was durch die oben zitierte Mitarbeiter/-innen-Befragung ermittelt werden konnte. Sie sind sich darüber im Klaren, welche Fähigkeiten bzw. Fertigkeiten sie bei den alltäglich auszuführenden Aufgaben unterstützen, und sind imstande, eventuellen diesbezüglichen Nachholbedarf zu definieren.

\section{Aktuelle Marktanforderungen - mögliche Konsequenzen für die philologischen Studiengänge}

Wie einleitend erläutert, suchen die meisten Germanistikabsolventen/-innen zurzeit ihr berufliches Glück als Spezialisten/-innen in verschiedenen Branchen der Wirtschaft. Die Kenntnis der deutschen - und in vielen Fällen 
obligatorisch der englischen - Sprache gilt dabei nicht als Selbstzweck, sondern als Mittel zum Zweck, indem komplexere Aufgaben- und Problemstellungen in einem internationalen Umfeld gemeistert werden können. Das konnte in dem vorliegenden Beitrag am Beispiel der Tätigkeit in einem Servicedesk gezeigt werden. Vor diesem Hintergrund sollen in einem nächsten Schritt mögliche Folgen diskutiert werden, welche die derzeitigen Marktanforderungen allgemein, sprich: die Erwartungen der modernen Dienstleistungszentren den arbeitssuchenden Germanisten/-innen gegenüber gegenwärtig für das Fach Germanistik bedeuten.

Vorausschickend sei betont, dass diese Diskussion mittlerweile zwar nicht neu ist, dass sie jedoch nicht an Aktualität eingebüßt hat. Im Großen und Ganzen lässt sie sich auf die Auseinandersetzung um die traditionelle Rolle der Universität in Zeiten eines raschen ökonomischen, gesellschaftlichen und bildungspolitischen Wandelns zurückführen, da Universitäten bereits seit Jahren nicht mehr als Institutionen angesehen werden, „mit denen man primär das Lehren und Lernen auf höchster Ebene assoziiert" (vgl. Heinemann 2001: 706), bzw. „als Bildungsstätten für intellektuelle Führungskräfte in allen Bereichen des Lebens" (ebd.). Aufgrund der Umsetzung der Bologna-Reform aus dem Jahr 1999 musste das herkömmliche Prinzip der Einheit von Forschung und Lehre insbesondere in den Bachelor-Studiengängen allmählich dem Slogan von Berufsqualifizierung weichen - die Universität der Gegenwart öffnete sich für das außerwissenschaftliche berufliche Umfeld (vgl. auch Grzeszczakowska-Pawlikowska 2012: 183ff.). In Bezug darauf lässt sich in Anlehnung an Wierlacher (2008: 25) von einem aktuell bestehenden gegenseitigen Verhältnis von Wissenschaft und Beruf sprechen, welches laut ihm vor allem als Anwendbarkeit (Brauchbarkeit) des universitär erworbenen Wissens begriffen wird. Die Praxisorientierung der universitären Ausbildung entspricht dabei einem der wesentlichsten Ziele des Bologna-Prozesses, nämlich dem der Beschäftigungsfähigkeit europäischer Akademiker/-innen (employability). Damit ist keine konkret definierte Berufstätigkeit gemeint. Anzustreben ist vielmehr die „Zukunftsfähigkeit [...] der wissenschaftlichen Ausgebildeten im Beschäftigungssystem" (ebd.: 32).

Angesichts der immer höher werdenden Ansprüche der Arbeitgeber/-innen an die Kompetenzen der Germanistikabsolventen/-innen im Moment ihres Einstiegs in den Wirtschaftssektor ist die Frage nach der gesellschaftlichen Relevanz einer klassischen (Auslands-)Germanistik im Sinne „wissenschaftlicher Qualität, philologischer Analyse und historischer Orientierung" (Hess-Lüttich 2009: 22) durchaus aktuell. Dies trifft umso mehr zu, als die gesunkene bzw. noch weiterhin sinkende Zahl der Bewerber/-innen für 
die germanistischen Studiengänge insbesondere für das Masterstudium schon jetzt ein reales Problem darstellt. Um den Anforderungen der Wirtschaftswelt an potenzielle Stellensuchende gerecht zu werden, sollten die Germanistikstudierenden zunächst eine fundierte sprachliche Ausbildung absolvieren. Diese Ausbildung sollte das Training von Perzeption, Produktion wie auch der breit angelegten Interaktion in der Fremdsprache Deutsch zum Ziel haben und unter Einbeziehung unterschiedlicher Textsorten, sowohl in Mündlichkeit als auch in Schriftlichkeit, sowie in Anlehnung an die Richtlinien des Gemeinsamen Europäischen Referenzrahmen für Sprachen erfolgen. Darüber hinaus sollte den Studierenden ebenfalls die Möglichkeit geboten werden, sich in verschiedenen Sachgebieten, wie etwa im Bereich von IT, Finanz- und Rechnungswesen, Buchhaltung etc., (fremd-)sprachlich zu sozialisieren. Die Vermittlung der einzelnen Fachsprachen sollte dabei weitgehend durch die Vermittlung von Sachwissen in den jeweiligen Bereichen begleitet bzw. ergänzt werden, sodass die zukünftigen Mitarbeiter/-innen eines BPO-, IT-/ITO- bzw. SSC-Centers nicht nur problemlos nach Übersetzungsäquivalenten greifen können, sondern auch jeweils wissen, was sich hinter den konkreten Fachbegriffen verbirgt. Mit anderen Worten: Das entsprechende Grundlagenwissen würde eine angemessene Handhabung der Fachtermini in verschiedenen Wirtschaftskontexten ermöglichen. Das Sprachvermögen im Deutschen bzw. in anderen Fremdsprachen, kurzum die sprachliche Kompetenz sollte also mit weiteren, fachbezogenen Kompetenzen einhergehen.

Schließlich verlangt der alltägliche Umgang mit Kunden, der grundsätzlich zum Aufgabenkern eines Servicedesks gehört, entsprechende Sozial- und Selbstkompetenz. Mit Sozialkompetenz sind nach Knauf (2001: 47) konkrete Kenntnisse, Fertigkeiten und Fähigkeiten zum situationsadäquaten Handeln in zwischenmenschlichen Beziehungen gemeint. Im Einzelnen kommt es dabei auf Kommunikations- und Kooperationsfähigkeit, Konfliktfähigkeit und Einfühlungsvermögen an. Bei Selbstkompetenz handelt es sich wiederum um Fähigkeiten und Einstellungen, in denen sich die individuelle Haltung zur Welt und insbesondere zur Arbeit ausdrückt. Es sind allgemeine Persönlichkeitseigenschaften wie etwa Flexibilität, Leistungsbereitschaft, Ausdauer, Zuverlässigkeit, Engagement und Motivation, die allerdings nicht nur im Arbeitsprozess von Bedeutung sind (vgl. ebd.). Die Sozial- und Selbstkompetenz stellen einen wesentlichen Bestandteil sog. Schlüsselkompetenzen bzw. sog. transversaler Kompetenzen dar (vgl. z.B. Gajewska 2016: 13), welche über konkrete Berufsqualifikationen, d.h. über reines Faktenwissen hinausgehen, da sie als eine effiziente Verbindung von Wissen, Können, Verstehen, Fähigkeiten, Erfahrungen, Haltungen, Einstellungen etc. fungieren (vgl. auch Klieme/Hartig 2007: 11ff.). 
Das Training von Schlüssel- bzw. transversalen Kompetenzen in einer Fremdsprache, wie zum Beispiel eine breit angelegte Kooperations- oder Konfliktfähigkeit als Grundlage für den optimalen Kommunikationsablauf, ist curricular bei weitem noch nicht gesichert. Dies gilt ebenfalls für die Vermittlung von rhetorischer Kompetenz als (Schlüssel-)Befähigung zum kommunikativen Handeln mit dem Ziel, intendierte soziale Interaktionen durch wirksames Miteinandersprechen (Gespräch) und Zueinanderreden (Rede) zu ermöglichen sowie die jeweiligen Kommunikationsprozesse zu reflektieren (vgl. Grzeszczakowska-Pawlikowska 2016: 188f.). Separate Seminare zur Rhetorizität verbaler, para- und nonverbaler Zeichen und zu deren kommunikativer Wirkung stellen in den germanistischen Studiengängen tatsächlich eine Ausnahme dar6. Bis diese Ausbildungslücke geschlossen wird, sollten die Germanistikstudierenden auf rhetorische Aspekte der mündlichen Kommunikation zumindest in den sprachpraktischen Lehrveranstaltungen systematisch aufmerksam gemacht werden (vgl. auch dieselbe 2015).

In dem vorliegenden Zusammenhang soll abschließend die große Relevanz der rhetorischen Kompetenz für die professionelle Telefonie, wie etwa ein Servicedesk, insbesondere in der interkulturellen Kommunikation hervorgehoben werden. Interessanterweise wurde in der oben aufgeführten Befragung explizit kein Schulungsbedarf in diesem Bereich angemeldet. Dies könnte wohl auf ein fehlendes bzw. unzureichendes Bewusstsein bezüglich der eigenen Effektivität im sprechsprachlichen Handeln als ServicedeskAgent/-in bzw. -Expert/-in zurückzuführen sein. Es scheint nämlich wenig wahrscheinlich, dass die befragten Mitarbeiter/-innen in den alltäglichen Telefongesprächen mit internationaler Kundschaft auf keine bzw. auf wenige Kommunikationsschwierigkeiten bzw. -störungen stoßen. Diese Annahme soll in gezielt angelegten empirischen Untersuchungen überprüft werden. Dabei soll davon ausgegangen werden, dass das Telefonieren in einer Fremdsprache nicht einfach auf Hörverständnis einerseits und sprachliche Korrektheit andererseits einzuschränken ist. Weitere Aspekte, wie die bereits erwähnte Kooperations- und Konfliktfähigkeit allgemein, ferner kulturell determinierte Ausdrucksformen von Höflichkeit, die Wirkung der Sprecherperson und deren Akzent sowie nicht zuletzt die Verständlichkeit von mündlichen Mitteilungen und die daraus resultierende gegenseitige Verständigung sind für die effiziente Kundenbetreuung und somit -zufriedenheit weitgehend entscheidend (ausführlicher zu dieser Problematik s. Hirschfeld/Neuber 2011). Ein separates Modul zum Handeln im Bereich

${ }^{6}$ Eine solche Ausnahme ist der germanistische Studiengang an der Universität Łódź, wo das Rhetorikseminar zum Studienprogramm (BA) gehört. 
der professionellen Telefonie, idealerweise in enger Zusammenarbeit mit den in Polen ansässigen Servicedesk-Zentren, wäre deshalb in den germanistischen Studiengängen sehr wünschenswert.

\section{Abschließende Bemerkungen}

Es soll in diesem Beitrag nicht bestritten werden, dass sich die ausländische bzw. fremdsprachige Germanistik (dazu s. ausführlicher Czachur 2012: 3ff.), wie es hier am Beispiel Polens ansatzweise problematisiert werden konnte, in einem Spannungsverhältnis zwischen Traditionsbewusstheit und Praxisorientierung befindet (vgl. auch Hess-Lüttich 2009: 22). Möchte man um jeden Preis den von außen kommenden Anspruch „beruflicher Nützlichkeit, praktischer Anwendbarkeit, zukunftsbezogener Offenheit" (ebd.) ignorieren, so würde man Gefahr laufen, dass das Fach Germanistik zukünftig zu einem „Orchideenfach“ bzw. aufgrund fehlenden Interesses, Deutsche Philologie im traditionellen Verständnis zu studieren, aus dem akademischen Bildungsangebot völlig gestrichen wird. Wegen der langen Tradition des Faches Deutsch als Fremdsprache sowie der langen Tradition der polnischen Germanistik überhaupt (vgl. z.B. Mihułka et al. 2016) wäre eine solche Entwicklung als außerordentlich großer Verlust zu werten, der vielleicht zukünftig auch nicht wieder gut zu machen wäre. Dieser Trend, die deutsche Sprache nicht (mehr) im eigenständigen Germanistikstudium, sondern im Rahmen von sog. German studies bzw. im Rahmen einer sog. School of foreign languages lediglich als ein Ausbildungsteil anzubieten, scheint gerade in Westeuropa im Kommen zu sein. Um diesem Trend entgegenzuwirken, sollte zunächst mit der Forderung des außeruniversitären Umfelds nach Praxisorientierung in der akademischen Bildung und der daraus resultierenden neuen Qualität von Universitäten der Gegenwart adäquat umgegangen werden. Die Wissenschaftlichkeit der Universität einerseits und die überall geforderte Beschäftigungsfähigkeit andererseits schließen sich nämlich meines Erachtens keinesfalls aus. Die Forderung nach employability darf vielmehr nicht als solche „nach einer unkritischen Übernahme vermeintlicher Forderungen der Wirtschaft nach Abkehr von Wissenschaftlichkeit" (Anz 2004: 5) interpretiert werden, wie das in der diesbezüglichen Diskussion ab und zu behauptet wird. Stattdessen soll an dieser Stelle erneut für eine mögliche Verschränkung von Wissenschaft und Praxisorientierung in der akademischen Bildung plädiert werden (vgl. auch GrzeszczakowskaPawlikowska 2012: 187f.), indem die Studienprogramme der Germanistik um anwendungs-/berufsbezogene Lehrveranstaltungen ergänzt werden, 
wie etwa Fachsprachenunterricht, Übersetzungs- und Dolmetschmodule, Gesprächs- und Redetrainings oder Seminare zu Kommunikationsmodellen etc. ${ }^{7}$ Es braucht dabei nicht auf traditionell bewährte philologische Fächer, wie etwa Literatur und -geschichte, Sprachgeschichte bzw. deutsche Sprachwissenschaft, verzichtet zu werden. Die Vermittlung dieser Fächer erfüllt auch heutzutage eine durchaus wichtige Rolle, da sie einen fundierten Ausgangspunkt für die Problematisierung der interkulturellen Kommunikation schafft (dazu vgl. mehr Hess-Lüttich 2009: 23ff.). Zugleich sollte in allen universitären Lehrveranstaltungen nach Möglichkeit systematisch die Vermittlung transversaler Kompetenzen erfolgen.

Die Öffnung der Universität der Gegenwart, somit auch der Germanistik für das wirtschaftliche Umfeld und die damit einhergehende Praxisbezogenheit mancher Lehrveranstaltungen ist meines Erachtens vor allem als eine Chance für das Fach zu betrachten. Und es handelt sich dabei nicht um die Vermarktung der germanistischen Studiengänge zwecks Gewinnung von Studierenden. Vielmehr eröffnet sich damit eine Chance für die Ausbreitung der empirischen Forschung auf neue Untersuchungsfelder, wie etwa Kommunikationsprozesse in der internationalen Wirtschaftswelt. Solche neuen Forschungserkenntnisse stellen zweifelsohne einen direkten Beitrag zur Optimierung von Verständigungsprozessen in einer Fremdsprache, hier im Deutschen, dar, welche ja über die Verwendung von Fachsprachen bzw. das Übersetzen/Dolmetschen weit hinausgehen.

Diese Öffnung stellt für manche (noch) traditionell/philologisch sozialisierten Hochschullehrer/-innen durchaus eine ernst zu nehmenden Schwierigkeit dar, da sie gewisse Ängste vor dem Verlust ihrer wissenschaftlichen Identität empfinden (könnten). In diesem Bereich wäre ein Umdenken nötig, welches aber seit Jahren allmählich fortschreitet (vgl. z.B. Zieliński 2010: 12ff.).

\section{Literaturverzeichnis}

- $\quad$ ABSL-Bericht (2017), Sektor nowoczesnych usług biznesowych $w$ Polsce, unter: http://g3.gazetaprawna.pl/p/_wspolne/pliki/2981000/2981561-raport-absl-2017.pdf (8.10.2018).

- $\quad$ ABSL-Bericht (2018), Sektor nowoczesnych usług biznesowych w Polsce, unter: http://absl.pl/wp-content/uploads/2018/06/raport_absl_2018_ PL_180527_epub-1.pdf (8.10.2018).

${ }^{7}$ Dies wird bereits seit Jahren den germanistischen Studiengängen an der Universität Łódź umgesetzt. 
- Anz Ch. (2004), „Beschäftigungsfähigkeit“ - Vereinbarkeit oder Konflikt mit Wissenschaftsorientierung? In: Benz W., Kohler J., Landfried K. (Hg.), Handbuch Qualität in Studium und Lehre, S. 1-11.

- Czachur W. (2012), Kulturwissenschaftliche Ausrichtung der kontrastiven Diskurslinguistik. Konsequenzen für die fremdsprachige Germanistik, „Zielsprache Deutsch“, 39 (3), S. 3-22.

- Gajewska E. (2016), Nauczyciel języka zawodowego tworzy własne dialogi dydaktyczne: od teorii do praktyki, „Języki Obce w Szkole“, 3, S. 12-18, unter: http://jows.pl/sites/default/files/gajewska_0.pdf (8.10.2018).

- Grzeszczakowska-Pawlikowska B. (2012), Einige Prämissen zur Sicherung der Attraktivität polnischer Germanistikabsolventen auf dem Arbeitsmerkt. In: Kaczmarek D., Makowski J., Michoń M., Weigt Z. (Hg.), Impulse für Forschung und Lehre, „Felder der Sprache Felder der Forschung. Lodzer Germanistikbeiträge“, Łódź: Primum Verbum, S. 183-197.

- Grzeszczakowska-Pawlikowska B. (2015), Ein integriertes Rhetoriktraining. Ansätze für germanistische Studiengänge in Polen, „Zeitschrift des Verbandes Polnischer Germanisten“, 4, S. 295-307.

- Grzeszczakowska-Pawlikowska B. (2016), Rhetorische Kompetenz bei polnischen Studierenden in der Fremdsprache Deutsch im universitären Kontext, „Studia Linguistica XXXV“ („Acta Universitatis Wratislaviensis 3742"), S. 193-210.

- Heinemann M. (2000), Textsorten des Bereichs Hochschule und Wissenschaft. In: Brinker K., Antos G., Heinemann W., Sager S. (Hg.), Text- und Gesprächslinguistik. Linguistics of Text and Conversation. Ein internationales Handbuch zeitgenössischer Forschung, Berlin, S. 702-709.

- Hess-Lüttich E.W.B. (2009), Wie kann man vom „Deutschen“ leben? Der Bedarf an Angewandter Germanistik und die Praxis Interkultureller Kommunikation - Ein Rückblick und Ausblick zur Einführung. In: Hess-Lüttich E.W.B. (Hg.), Wie kann man vom „Deutschen“ leben? Zur Praxisrelevanz der interkulturellen Germanistik, "Cross Cultural Communication“, Bd. 17, Frankfurt am Main: Peter Lang, S. 19-45.

- Hirschfeld U., Neuber B. (2011), Erforschung und Optimierung der Callcenterkommunikation, Berlin: Frank \& Timme.

- $\quad$ Klieme E., Hartig J. (2007), Kompetenzkonzepte in den Sozialwissenschaften und im erziehungswissenschaftlichen Diskurs, „Zeitschrift für Erziehungswissenshaften", 10, Sonderheft 8, S. 11-29.

- Knauf H. (2001), Schlüsselqualifikationen, „HSW“, 2, S. 45-49, unter: http://www.helen-knauf.de/wp-content/uploads/2011/12/Knauf_ Schluesselkompetenzen_2001.pdf (8.10.2018). 
- Makowski J. (2018), Rola czynników obiektywnych $w$ dydaktyce języków specjalistycznych na poziomie uniwersyteckim. Omówienie wyników badania ankietowego w ramach projektu "Językowy baromter Łodzi“, „Komunikacja Specjalistyczna“, 16 (im Druck).

- Makowski J. (in diesem Band), Zur textuellen Teilkompetenz im arbeitsmarktorientierten Fachsprachenunterricht am Beispiel der Branche moderner Unternehmensdienstleistungen und des Wissensbereichs Finanzen.

- Mihułka K., Sieradzka M., Budziak R. (2016), Die Fremdsprache Deutsch in Polen: Anfänge, Gegenwart, Perspektiven, Rzeszów: Wydawnictwo Uniwersytetu Rzeszowskiego.

- Wassermann J. (2008), Business Process Outsourcing - Strategische Handlungsoptionen vor dem Hintergrund der Industrialisierung im Bankund Versicherungsgewerbe, Saarbrücken: Verlag Dr. Müller.

- Wierlacher A. (2008), Zur partiellen Verschränkung von wissenschaftlicher und beruflicher Bildung. In: Arbeitskreis für interkulturelle Germanistik in China: Deutsch-chinesisches Forum Interkultureller Bildung, Bd. 1: Wissenschaftskommunikation im internationalen Kontext, München: Iudicium, S. 25-43.

- Włodarczyk M. (2016), Jakie procesy kryją się pod popularnymi skrótami BPO, SSC, ITO?, unter: http://www.outsourcingportal.eu/pl/jakie-procesy-kryja-sie-pod-popularnymi-skrotami-bpo-ssc-ito (8.10.2018).

- Zieliński L. (2010), Angewandte Germanistik - Notwendigkeit oder Chance, „Studia Germanica Gedanensia“, 23, S. 11-21.

\section{Graduates of Germam studies at the service desk - opportunities or difficulties for the subject?}

The training of German teachers at Polish universities is no longer as self-evident as it was in the 1990s - ever fewer students are taking courses in German as a Foreign Language.

There are many reasons for this trend: on the one hand the hegemony of English as a global language, the decline in the demand for teachers of German and the corresponding demographic low; on the other, the miserable pay conditions for Polish teachers and comparatively high salaries in the business sector.

It is therefore not surprising that an increasing number of university students of German wish to develop careers as specialists in various economic sectors. This is especially the case as the demand for employees with a knowledge 
of German is currently particularly high. This raises the question of what the employment market means in concrete terms for degree programmes in German at Polish Universities. Should prevailing market conditions be seen as an opportunity to reorientate a subject that is currently attempting to protect established disciplinary norms or do they rather constitute a challenge, for example to (still) traditionally socialised university lecturers employed in arts and humanities disciplines? This paper will explore these questions.

Keywords: economic sectors, German as a foreign language, service-desk 